\title{
Biomimetic reactions in conducting polymers for artificial muscles: sensing working conditions
}

\author{
Victor H. Pascual*a, Toribio F. Otero ${ }^{\mathrm{a}}$, J. Schumacher ${ }^{\mathrm{b}}$ \\ ${ }^{a}$ Laboratory of Electrochemistry and Intelligent Materials, Technical University of Cartagena, ETSII, \\ Campus Alfonso XIII, 30203, Cartagena, Spain.; ' Arquimea Ingeniería, Margarita Salas 10, 28919, \\ Leganés, Spain.
}

\begin{abstract}
In the dense gel that is the intracellular matrix forming part of living cells electrochemical reactions take place provoking the interchange of ions and water with the surroundings. Systems containing conducting polymers mimic this feature of biological organs. In particular, conducting polymers are being studied as dual sensing-actuating reactive materials giving new multifunctional sensing-actuators, which allow the construction and theoretical description of artificial proprioceptive devices. Here films of polypyrrole/dodecyl benzene sulfonate (PPy-DBS) coating a platinum electrode were submitted to potential sweeps at different sweep rates in order to explore if the polymer reaction senses the working electrochemical conditions. The effective consumed electrical energy per cycle follows a fast decrease when the scan rate increases described by the addition of two exponential sensing functions. Moreover, the variation of the hysteresis from the parallel charge/potential loop with the scan rate is also described by the addition of two exponential functions. In both cases the exponential functions fitting results at low scan rates are related to reaction-driven conformational movements of the polymer chains, being closer to biochemical conformational and allosteric sensors. The second exponential functions fitting results at high scan rates are related to diffusion kinetic control, being closer to present electrochemical sensors.
\end{abstract}

Keywords: Conducting polymers, Biochemical sensors, Electro-chemo-biomimesis

\section{INTRODUCTION}

There are organs in the living beings able to sense the conditions under which they are working, existing a constant feedback communication between them and the brain, ultimately causing proprioception ${ }^{1-3}$.

From the last decades of the past century, multiple devices based on molecular motors working due to the action of electrochemical reactions have emerged. They are constituted by electroactive materials such as conducting polymers or carbon nanotubes ${ }^{4}$. Those electrochemical reactions, like those taking place in living cells, lead to an exchange of solvent and ions with the medium in which they are immersed ${ }^{5}$. Through conformational motions of the reactive chains, these reactions generate or destroy the necessary free room to lodge or expel counterions and solvent. By means of this phenomenon a reversible change of the material composition is provoked, enabling the creation of biomimetic devices such as artificial muscles ${ }^{6-8}$, nervous interfaces ${ }^{9,10}$ or sensors $^{11-13}$.

The electrical energy $(U)$ consumed by any electrochemical reaction depends on the consumed charge $(Q)$ and the electric potential $(E)$ :

$$
U=Q E
$$

Being $E$ a function of the working energetic conditions ${ }^{3}$, it is revealed that there are, in fact, several sensors and one actuator working at the same time in a physically uniform device, exposing how a device working under these principles would only need two connecting wires for sensing and actuating functions.

In this work the sensing properties of the reaction have been studied by using polypyrrole/dodecylbenzene sulfonate (PPy-DBS) films, in particular, their ability to sense changes in the electrochemical energy available when the scan rate of potential sweeps is modified. When other sources of energy (electrolyte concentration, temperature, pressure and mechanical conditions) are keep at constant values the effective electrochemical energy $(\Delta u)$ applied to the polymeric film depends on the scan rate $(v)$ as follows ${ }^{14,15}$

Bioinspiration, Biomimetics, and Bioreplication 2017, edited by Mato Knez, Akhlesh Lakhtakia, Raúl J. Martín-Palma Proc. of SPIE Vol. 10162, 101620D - (c) 2017 SPIE · CCC code: 0277-786X/17/\$18 · doi: 10.1117/12.2259912 


$$
\Delta u=\frac{\Delta U(U)}{v\left(V s^{-1}\right)}=\Delta Q(C) \frac{\Delta E(V)}{v\left(V s^{-1}\right)}=\frac{\Delta Q \cdot \Delta E}{v}(C s)
$$

\section{METHODOLOGY}

\subsection{Chemicals and Equipment}

To generate the films, the next products were used: distilled pyrrole (Fluka), dodecylbenzene sulfonate acid (HDBS) solution (Sigma-Aldrich), sodium perchlorate (Sigma-Aldrich) and Milli-Q water.

For the electrochemical experiments and polymerization process an Autolab potentiostat/galvanostat PGSTAT302 (NOVA 1.11 software) was employed, as well as an electrochemical cell with a three electrode configuration using a stainless steel plate (total immerse area of 2,52 $\mathrm{cm}^{2}$ ) as counter electrode $(\mathrm{CE})$ and a platinum sheet (total immerse area of $\left.1 \mathrm{~cm}^{2}\right)$ as working electrodes (WE) and an $\mathrm{Ag} / \mathrm{AgCl}(3 \mathrm{M} \mathrm{KCl})$ reference electrode (RE, Metrohm).

Masses were determined by using a Sartorious Extended ED224s balance.

\subsection{Film Preparation}

Ppy-DBS films were synthesized at room temperature over the platinum sheet from a $0.2 \mathrm{M}$ DBS and $0.2 \mathrm{M}$ pyrrole aqueous solution though the application of an anodic current density of $2 \mathrm{~mA} \mathrm{~cm} \mathrm{c}^{-2}$ for $500 \mathrm{~s}$. Being the mass of the rinsed film of $9 \mathrm{mg}$.

\subsection{Characterization Methodologies}

Once electrogenerated, the films were subjected to sweep potentials between -1000 and $100 \mathrm{mV}$ at a scan rate of $30 \mathrm{mVs}$ 1 in a $0.1 \mathrm{M}$ NaClO 4 aqueous solution up to obtaining stationary voltammetric responses from consecutive cycles. This
fact allows to be sure any material memory from the generation process has been erased ${ }^{2}$. After this the influence of the scan rate was analyzed.

The film used to attain the experimental results must be always the same, that is, it has to show the same oxidation/reduction behavior, same electroactivity and same consumed oxidation reduction charge when identical control conditions are applied. These controls consisted of cycling the film under the same conditions expressed in the previous paragraph. When the charge deviation was over 5\% if compared with the other controls the film was discard and a new one electrogenerated.

\section{RESULTS}

\subsection{Voltammetric responses}

PPy-DBS films in a $\mathrm{NaClO}_{4}$ aqueous solution and subjected to potential sweeps show a reversible oxidation/reduction process of the polypyrrole chains leading into an exchange of cations and solvent for charge and osmotic balance, respectively, according to the reaction ${ }^{21,22}$

$$
\left[(P P y)_{S}\left(D B S^{-} N a^{+}\right)_{n}\left(H_{2} 0\right)_{m}\right] \rightleftharpoons\left(P P y^{n+}\right)\left(D B S^{-}\right)_{n}+n N a^{+}+m\left(H_{2} 0\right)+n e^{-}
$$

Being PPy the active centers in the polymer that will accommodate a positive charge after oxidation, $D B S$ the anion that was trapped during the synthesis in the film and $\mathrm{Na}^{+}$the exchanged cation during the redox process.

With a potential sweep slow enough and a monodisperse polymeric film (all the constitutive polypyrrole chains have the same conjugated length) the reaction takes place in $n$ consecutive equilibrium steps. 
In Figure 1 the stationary voltammetric responses are displayed. During oxidation (Equation 3 forward) electrons are removed from the PPy chains, leading to an expulsion of $\mathrm{Na}^{+}$cations and water molecules for charge and osmotic balance and shrinking the film in the process. When electrons are injected to the PPy chains, reducing the film (Equation 3 backward). Now the sodium cations come from the solution into the film, bringing water molecules with them for charge and osmotic balance and swelling the film ${ }^{23}$.

In Figure 1 it can be appreciated a strong shift of the oxidation and reduction maximum peaks towards more anodic and cathodic values, respectively increasing the electrochemical hysteresis (potential difference between the anodic and the cathodic maxima).
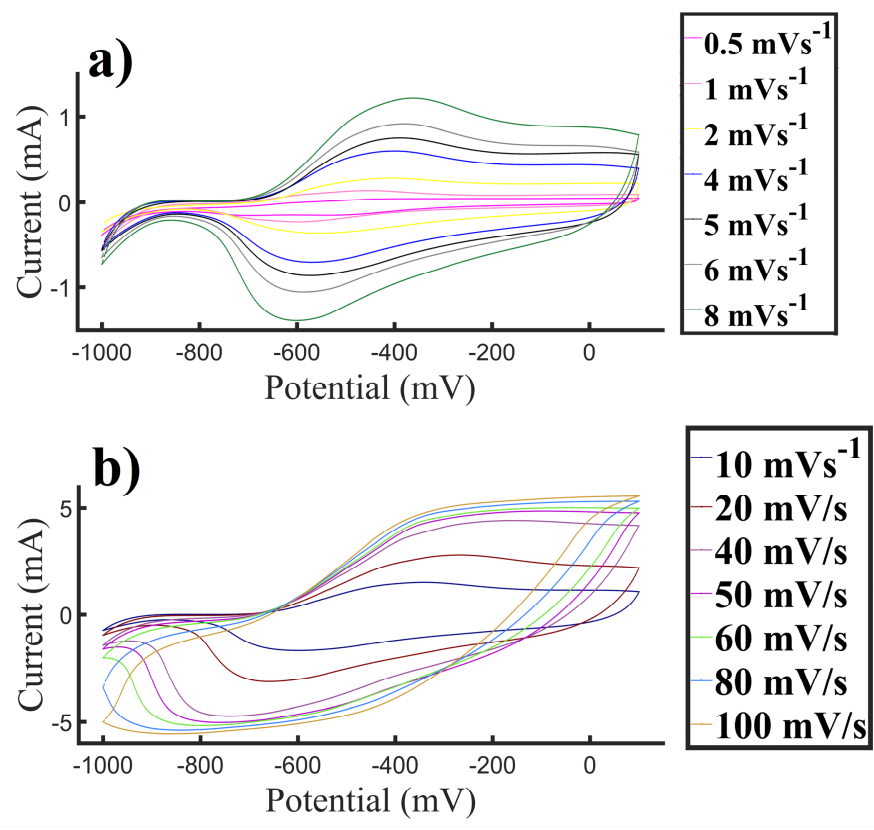

Figure 1. Stationary voltammetric responses of a PPy-DBS film coating a platinum electrode between the potential limits of ($1000,100) \mathrm{mV}$ in $0.1 \mathrm{M} \mathrm{NaClO}_{4}$ aqueous solution. (a) from 0.5 to $8 \mathrm{mVs}^{-1}$, (b) from 10 to $100 \mathrm{mVs}^{-1}$.

\subsection{Coulovoltammetric responses}

Integrating the voltammetric responses shown in Figure 1 the coulovoltammetric (Q-E) responses can be depicted (Figure 2 ). In this case the potentiostat-galvanostat used provides this response automatically.

Figure 2 shows some representative coulovoltammetric responses corresponding to the voltammetric responses of Figure 1. Positive charge increments represent oxidation charges while negative ones express reduction charges.

All responses exhibit a close loop on their right side representing the reversible charge consumed during a redox process (Equation 3) and an open part on the left quantifying the charge owing to irreversible reduction reactions at cathodic potentials simultaneously to that corresponding to the polymer reversible reduction ${ }^{24}$. 

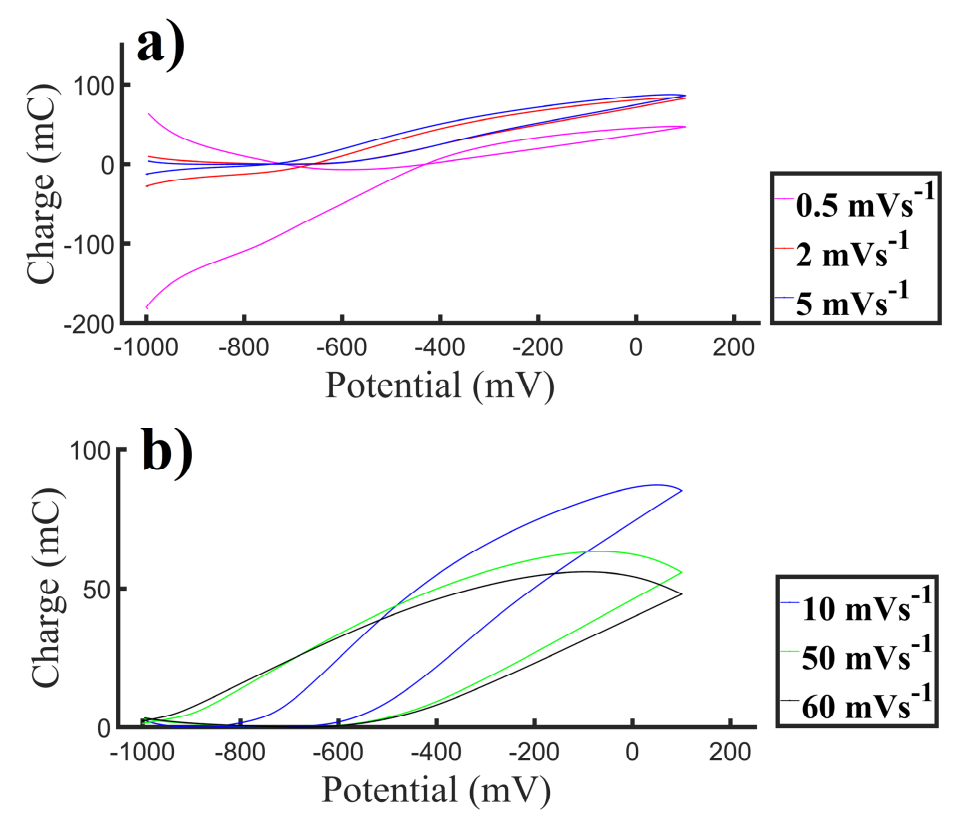

Figure 2. Representative stationary coulovoltammetric responses of a PPy-DBS film coating a platinum electrode between the potential limits of $(-1000,100) \mathrm{mV}$ in $0.1 \mathrm{M} \mathrm{NaClO4}$ aqueous solution attained by integration of the corresponding voltammetric responses of Figure 1. (a) from $0.5,2$ and $5 \mathrm{mVs}-1$, (b) 10,50 and $60 \mathrm{mVs}^{-1}$.

Figure 4 shows both the reversible and the irreversible charge evolution with the scan rate in the studied potential range. The irreversible charge always decreases when the scan rate increases. This is a contribution that has been associated with the hydrogen evolution originated in its most part in the platinum polymer interface but also with a small contribution coming from the HDBS component of the film ${ }^{23,25,26}$. This evolution is limited by the water diffusion rate through the full film thickness and since the polymeric chains remain more shrunken when the scan rate increases, the water diffusion coefficient decreases and less hydrogen evolves for the highest scan rates ${ }^{27}$.

Below $8 \mathrm{mVs}^{-1}$ the reversible charge of the oxidation / reduction process increases with the scan rate and decreases after this value. The origin of the maximum is in a degradation process of the film that increases with lower scan rates.

According to the Electrochemically Stimulated Conformational Relaxation (ESCR) model. Below $8 \mathrm{mVs}^{-1}$ there is a kinetic control of the conformational processes while over that value the process is control by diffusion ${ }^{27}$.

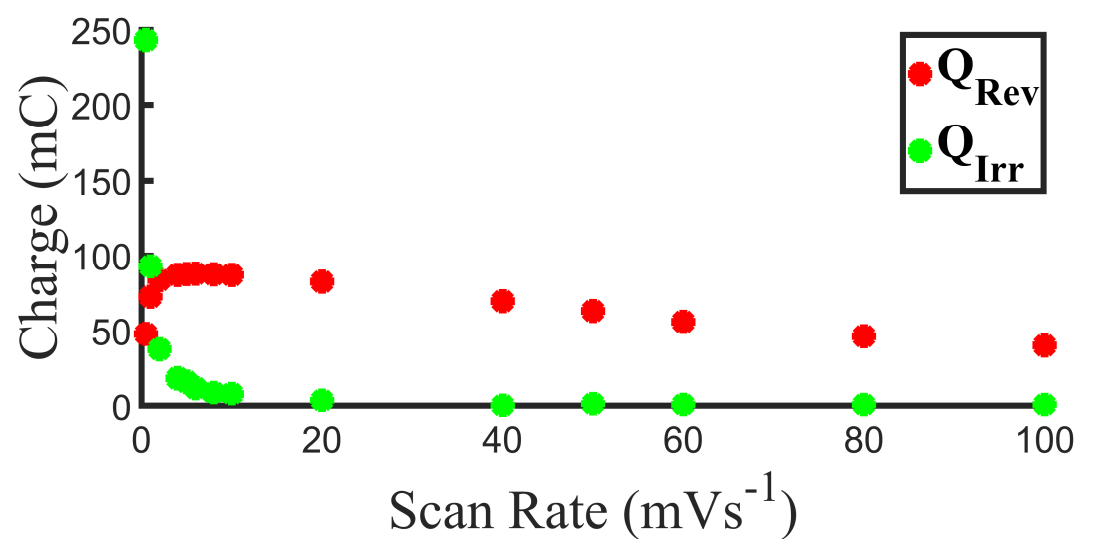

Figure 3. Evolution of the consumed charge with the scan rate. Reversible charge red dots and irreversible charge green dots. 


\subsection{Energy}

In Figure 4 the evolution of the effective electrical energy consumed (Equation 2) by the reversible oxidation/reduction of the film at different scan rates is depicted and it fits to the equation

$$
u_{r e v}=a e^{b v}+c e^{d v}
$$

Where $a=1.2010^{5} \mathrm{~mJ} \mathrm{mV}^{-1} \mathrm{~s}, b=-0.73 \mathrm{mC}^{-1}, c=2.3710^{4} \mathrm{~mJ} \mathrm{mV}^{-1} \mathrm{~s}$ and $d=-0.08 \mathrm{mC}^{-1}$, being the correlation coefficient, $r^{2}: 0.99$.

This evolution fitting two exponential functions is predicted by the ESCR model. One is related to the conformational compaction/relaxation of the polymer chains (molecular motors) and the other to the counterion diffusion through the film. Just for the lower scan rates there is time to get deep oxidation states followed by the corresponding reduction.

The important consequence of this is the effective electrical energy is a function of the scan rate, that is, of the working electrochemical conditions or in other words this magnitude is sensing the available energy.

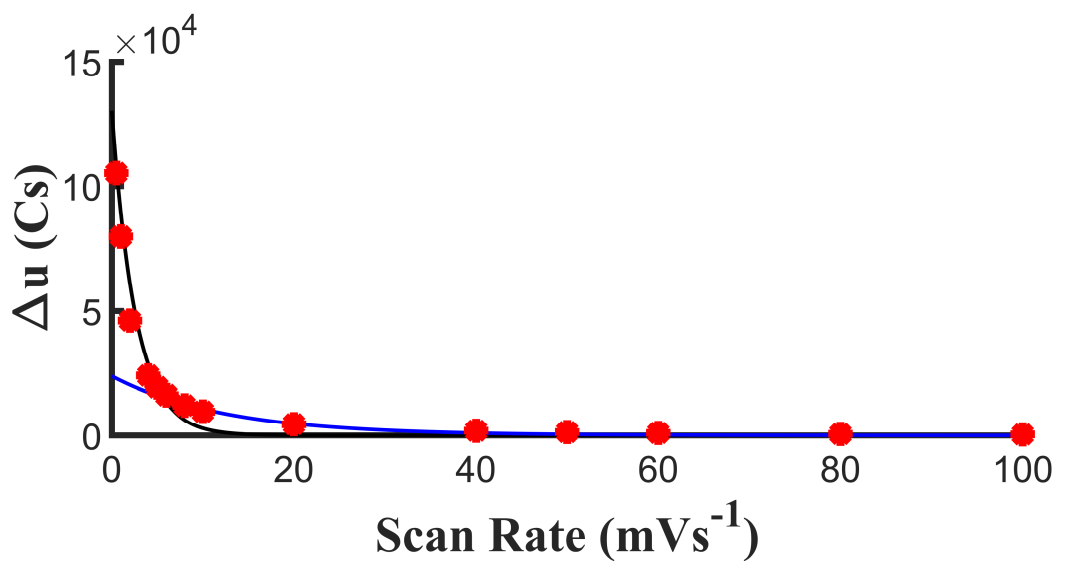

Figure 4. Effective consumed energy vs scan rate

\subsection{Hysteresis Potential Gradient}

Figure 5 represents the hysteresis of the $Q-E$ loops, which is a way of seeing how the conformational motions create structural changes (swelling or shrinking) taking the electrochemical response out of the equilibrium during the potential sweep ${ }^{23,28}$. Without this effect that retards the counterions and solvent molecules it would be expected that the system would be in equilibrium and none hysteresis would appear on the coulovoltammetric responses.

The evolution of this hysteresis with the scan rate fits the equation

$$
\Delta E=a e^{b \nu}+c e^{d v}
$$

Where $a=517.1 \mathrm{mV}, b=0.0018 \mathrm{~s}, c=-392.4 \mathrm{mV}$ and $d=-0.030 \mathrm{~s}$, being the correlation coefficient $r^{2}=0.99$.

There is again the addition of two exponentials, evolution that indicates the control at lower scan rates of conformational relaxation/compaction processes and a diffusion kinetic control for the higher ones. And another magnitude (potential hysteresis) that senses working condition has been found. 

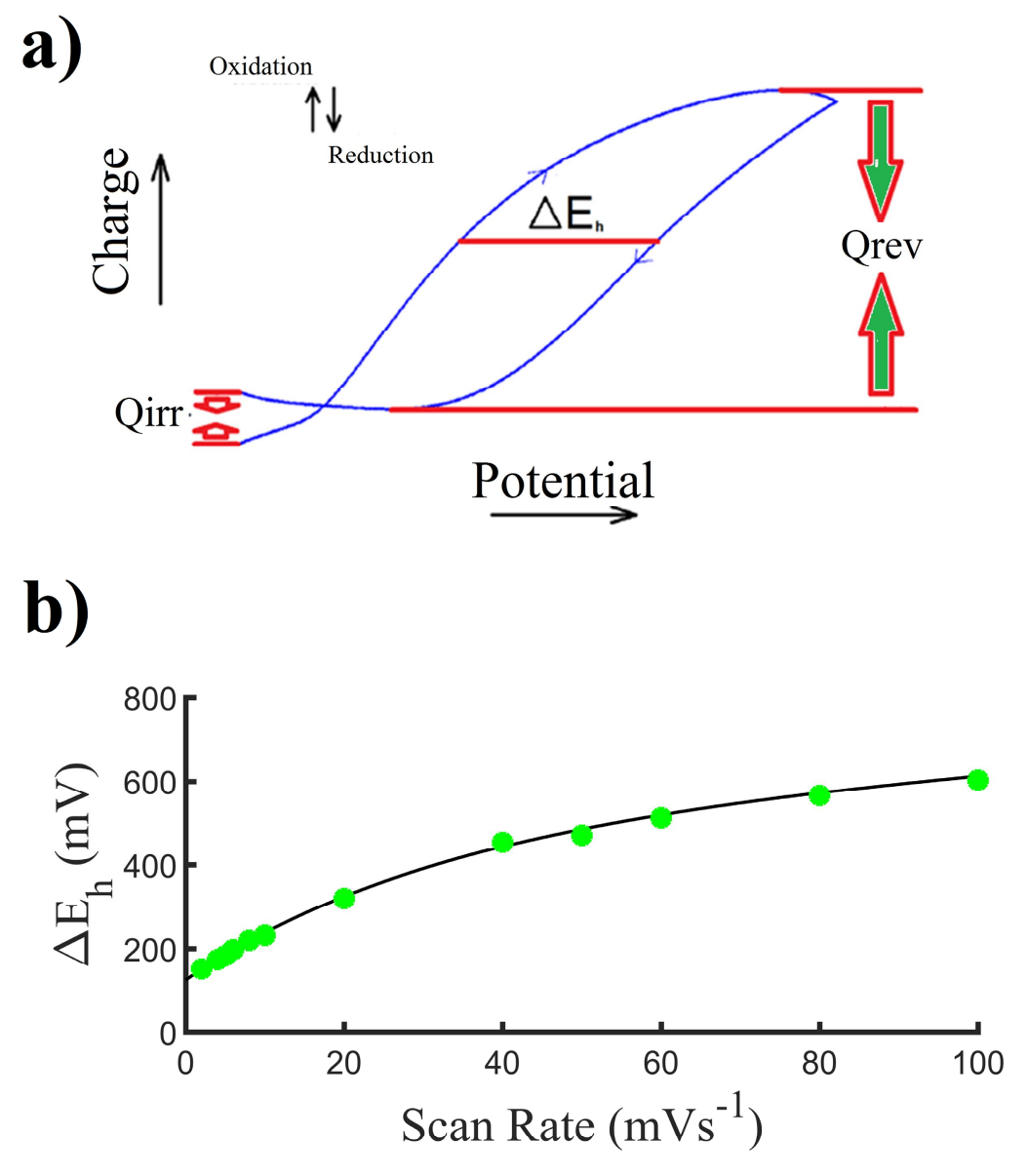

Figure 5. (a) Coulovoltammetric response from the PPy-DBS film at $10 \mathrm{mVs}^{-1}$ in $0.1 \mathrm{M} \mathrm{NaClO4}$ aqueous solution: ( $\left.\mathrm{Q}_{\mathrm{rev}}\right)$ is the reversible charge related film oxidation/reduction, $\left(Q_{\text {irr }}\right)$ is the irreversible charge related to the hydrogen evolution and $\left(\Delta E_{h y s}\right)$ quantifies the hysteresis potential gradient. (b) Hysteresis potential gradient calculated as indicated in figure a for different scan rates between 0,5 and $100 \mathrm{mVs}^{-1}$.

\section{DISCUSSION AND CONCLUSIONS}

Two magnitudes sensing the electrochemical working conditions have been found: the effective electrical energy consumed and the coulovoltammetric hysteresis. Both of them fit, empirically, to the addition of two exponential functions.

One of the exponential functions is associated to the kinetic control of reaction driven conformational motions, which dominate at the lowest scan rates (below $8 \mathrm{mVs}^{-1}$ ). The other one is related to the kinetic control of counterions in the film and it prevails at the highest scan rates $\left(\right.$ above $\left.8 \mathrm{mVs}^{-1}\right)$.

The first one is similar to the biological sensors based on the conformational and allosteric movements of enzymes, while the second one is close to galvanostatic sensors.

Any electrochemical device of PPy-DBS and probably constituted by any electroactive material will detect during actuation any variation in the available electrochemical energy, that is, it will sense the working conditions. Enabling the theoretical description of dual sensing-actuating devices ${ }^{3,29,30}$ that actually mimic biological organs. 


\section{ACKNOWLEDGEMENT}

The research was supported by European Union's Horizon 2020 research and innovation program under the Marie Sklodowska-Curie grant agreement No 641822 .

\section{REFERENCES}

[1] Ashton-Miller, J. A., Wojtys, E. M., Huston, L. J.., Fry-Welch, D., "Can proprioception really be improved by exercises?,” Knee Surg. Sports Traumatol. Arthrosc. 9(3), 128-136 (2001).

[2] Lackner, J. R.., DiZio, P., "Vestibular, proprioceptive, and haptic contributions to spatial orientation," [Annual Review of Psychology], Annual Reviews, Palo Alto, 115-147 (2005).

[3] Otero, T. F.., Martinez, J. G., "Physical and chemical awareness from sensing polymeric artificial muscles. Experiments and modeling," Prog. Polym. Sci. 44, 62-78 (2015).

[4] Otero, T. F., "Biomimetic Conducting Polymers: Synthesis, Materials, Properties, Functions, and Devices," Polym. Rev. 53(3), 311-351 (2013).

[5] Otero, T. F.., Martinez, J. G., "Biomimetic intracellular matrix (ICM) materials, properties and functions. Full integration of actuators and sensors," J. Mater. Chem. B 1(1), 26-38 (2013).

[6] Mirfakhrai, T., Madden, J. D. W.., Baughman, R. H., "Polymer artificial muscles," Mater. Today 10(4), 30-38 (2007).

[7] Otero, T., Angulo, E., Rodriguez, J.., Santamaria, C., "Electrochemomechanical Properties from a Bilayer - Polypyrrole Nonconducting and Flexible Material Artificial Muscle,” J. Electroanal. Chem. 341(1-2), 369-375 (1992).

[8] Pei, Q.., Inganas, O., "Conjugated Polymers and the Bending Cantilever Method - Electrical Muscles and Smart Devices," Adv. Mater. 4(4), 277-278 (1992).

[9] Asplund, M., Nyberg, T.., Inganas, O., "Electroactive polymers for neural interfaces," Polym. Chem. 1(9), 1374-1391 (2010).

[10] Green, R.., Abidian, M. R., “Conducting Polymers for Neural Prosthetic and Neural Interface Applications,” Adv. Mater. 27(46), 7620-7637 (2015).

[11] Dhand, C., Das, M., Datta, M.., Malhotra, B. D., "Recent advances in polyaniline based biosensors," Biosens. Bioelectron. 26(6), 2811-2821 (2011).

[12] Gerard, M., Chaubey, A.., Malhotra, B. D., “Application of conducting polymers to biosensors,” Biosens. Bioelectron. 17(5), 345-359 (2002).

[13] Gupta, N., Sharma, S., Mir, I. A.., Kumar, D., “Advances in sensors based on conducting polymers,” J. Sci. Ind. Res. 65(7), 549-557 (2006).

[14] Grande, H.., Otero, T. F., "Intrinsic asymmetry, hysteresis, and conformational relaxation during redox switching in polypyrrole: A coulovoltametric study,” J. Phys. Chem. B 102(39), 7535-7540 (1998).

[15] Otero, T. F., Grande, H.., Rodriguez, J., "Role of conformational relaxation on the voltammetric behavior of polypyrrole. Experiments and mathematical model," J. Phys. Chem. B 101(42), 8525-8533 (1997).

[16] Barbero, C., Kotz, R., Kalaji, M., Nyholm, L.., Peter, L., "Ion-Exchange and Memory Effects in Polyaniline," Synth. Met. 55(2-3), 1545-1551 (1993).

[17] Goldenberg, L., Aeiyach, S.., Lacaze, P., "Observation of a Memory Effect in the Case of Poly(p-Phenylene), Synthesized by Electrochemical Oxidation of Benzene in a Superacid Medium," Synth. Met. 55(2-3), 1365-1370 (1993).

[18] Heinze, J.., Rasche, A., "The memory effect in solution," J. Solid State Electrochem. 10(3), 148-156 (2006).

[19] Villeret, B.., Nechtschein, M., "Memory Effects in Conducting Polymers," Phys. Rev. Lett. 63(12), 1285-1287 (1989).

[20] Vorotyntsev, M. A., Skompska, M., Pousson, E., Goux, J.., Moise, C., "Memory effects in functionalized conducting polymer films: titanocene derivatized polypyrrole in contact with THF solutions," J. Electroanal. Chem. 552, 307-317 (2003).

[21] Otero, T. F., Martinez, J. G.., Arias-Pardilla, J., "Biomimetic electrochemistry from conducting polymers. A review Artificial muscles, smart membranes, smart drug delivery and computer/neuron interfaces," Electrochimica Acta 84, 112-128 (2012).

[22] Valero Conzuelo, L., Arias-Pardilla, J., Cauich-Rodriguez, J. V., Afra Smit, M.., Fernandez Otero, T., "Sensing and Tactile Artificial Muscles from Reactive Materials," Sensors 10(4), 2638-2674 (2010).

[23] Otero, T. F., Martinez, J. G., Fuchiwaki, M.., Valero, L., "Structural Electrochemistry from Freestanding Polypyrrole Films: Full Hydrogen Inhibition from Aqueous Solutions," Adv. Funct. Mater. 24(9), 1265-1274 (2014).

[24] Otero, T. F., Alfaro, M., Martinez, V., Perez, M. A.., Martinez, J. G., "Biomimetic Structural Electrochemistry from Conducting Polymers: Processes, Charges, and Energies. Coulovoltammetric Results from Films on Metals Revisited,” Adv. Funct. Mater. 23(31), 3929-3940 (2013).

[25] Fuchiwaki, M.., Otero, T. F., "Polypyrrole-para-phenolsulfonic acid/tape artificial muscle as a tool to clarify biomimetic driven reactions and ionic exchanges," J. Mater. Chem. B 2(14), 1954-1965 (2014).

[26] Valero, L., Martinez, J. G.., Otero, T. F., "Creeping and structural effects in Faradaic artificial muscles," J. Solid State Electrochem. 19(9), 2683-2689 (2015). 
[27] Suarez, I. J., Otero, T. F.., Marquez, M., "Diffusion coefficients in swelling polypyrrole: ESCR and Cottrell models,” J. Phys. Chem. B 109(5), 1723-1729 (2005).

[28] Fuchiwaki, M., Martinez, J. G.., Otero, T. F., “Asymmetric Bilayer Muscles. Cooperative and Antagonist Actuation,” Electrochimica Acta 195, 9-18 (2016).

[29] Martinez, J. G.., Otero, T. F., "Mechanical awareness from sensing artificial muscles: Experiments and modeling,” Sens. Actuators B-Chem. 195, 365-372 (2014).

[30] Otero, T. F., Sanchez, J. J.., Martinez, J. G., "Biomimetic Dual Sensing-Actuators Based on Conducting Polymers. Galvanostatic Theoretical Model for Actuators Sensing Temperature," J. Phys. Chem. B 116(17), 5279-5290 (2012). 